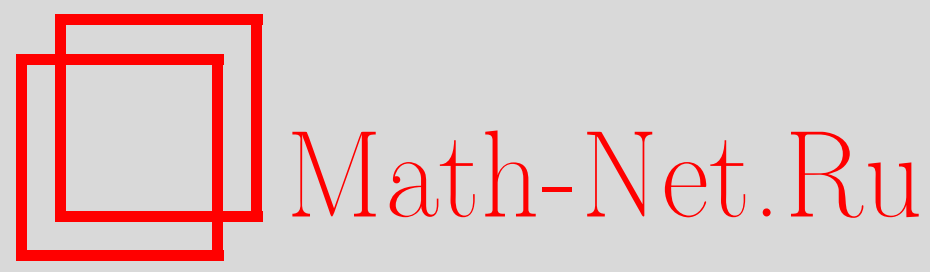

Ю. М. Воробьев, О линеаризации гамильтоновых систем на пуассоновых многообразиях, Матем. заметки, 2005, том 78, выпуск 3, 323-330

DOI: https://doi.org/10.4213/mzm2601

Использование Общероссийского математического портала Math-Net.Ru подразумевает, что вы прочитали и согласны с пользовательским соглашением http://www . mathnet.ru/rus/agreement

Параметры загрузки:

IP : 54.89 .56 .158

26 апреля 2023 г., 16:18:41

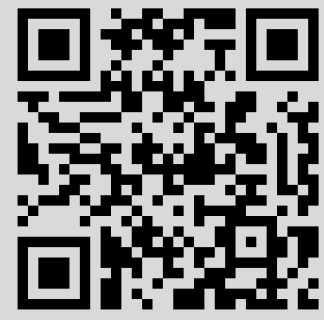




\title{
О ЛИНЕАРИЗАЦИИ ГАМИЛЬТОНОВЫХ СИСТЕМ НА ПУАССОНОВЫХ МНОГООБРАЗИЯХ
}

\section{Ю. М. Воробьев}

\begin{abstract}
Линеаризация гамиль тоновой системы на пуассоновом многообразии вдоль данного (сингулярного) симплектического листа определяет некую динамическую систему на нормальном расслоении к листу, которая назьвается системой в вариациях. Показано, что система в вариациях допускает согласованную гамильтонову структуру, если существует трансверсаль к листу, которая инварианта относительно фазового потока исходной системы. В случае, когда трансверсальная алгебра Ли симплектического листа является полупростой, это условие оказьвается также необходимьм.
\end{abstract}

Библиография: 10 названий.

1. Введение. Пусть имеется пуассоново многообразие $(M, \Psi)$ со скобкой Пуассона

$$
\{F, G\}_{\Psi}=\Psi(d F, d G)=\Psi^{J I}(y) \frac{\partial F}{\partial y^{I}} \frac{\partial G}{\partial y^{J}}
$$

(здесь и далее суммирование проводится по повторяющимся индексам). Пусть задана гамильтонова система $(M, \Psi, H)$, соответствующая гамильтонову векторному полю

$$
X_{H}=\Psi^{\natural} d H=-\Psi^{J I}(y) \frac{\partial H}{\partial y^{I}} \frac{\partial}{\partial y^{J}} ;
$$

здесь $\Psi^{\natural}: T^{*} M \rightarrow T M-$ морфизм векторных расслоений, индуцированньй тензором Пуассона $\Psi$. В соответствии с общей схемой $[1]$, [2], процедура линеаризации для динамической системы $\left(M, X_{H}\right)$ задает векторное поле $\operatorname{Var}\left(X_{H}\right)$ на касательном расслоении $T M$. Динамическая система, соответствующая $\operatorname{Var}\left(X_{H}\right)$, называется системой в вариациях векторного поля $X_{H}$ на $T M$. В локальных координатах $\left(y^{I}, u^{J}\right)$ на $T M$ система в вариациях имеет вид

$$
\begin{aligned}
\dot{y}^{I} & =-\Psi^{J I}(y) \frac{\partial H}{\partial y^{I}} \\
\dot{u}^{J} & =-\left(\frac{\partial \Psi^{J S}(y)}{\partial y^{I}} \frac{\partial H}{\partial y^{S}}+\Psi^{J S} \frac{\partial^{2} H}{\partial y^{S} \partial y^{I}}\right) u^{I} .
\end{aligned}
$$

Работа выполнена при частичной поддержке программы CONACYT, грант № 35212-Е. 
Инвариантное определение векторного поля $\operatorname{Var}\left(X_{H}\right)$ дается с помощью канонической инволюции на $T T M[1]$. Как известно, система $(1.1),(1.2)$ является гамильтоновой относительно тангенциальной пуассоновой структуры на $T M[1]$.

Наша цель - изучение линеаризованной гамильтоновой динамики на заданном (сингулярном) симплектическом листе. Пусть задан замкнутый симплектический лист $(B, \omega)$ пуассонова многообразия $(M, \Psi)$ с симплектической структурой

$$
\omega=\frac{1}{2} \omega_{i j}(\xi) d \xi^{i} \wedge d \xi^{j}
$$

Ограничение $X_{H}$ на $B$ является гамильтоновым векторным полем на $(B, \omega)$,

$$
v_{f}=\left.X_{H}\right|_{B}=\omega^{i s}(\xi) \frac{\partial f}{\partial \xi^{s}},
$$

где $f=\left.H\right|_{B}$. Пусть $T_{B} M$ - ограничение касательного расслоения $T M$ на лист $B$. Hopмальным расслоением $E=T_{B} M / T B$ к листу $B$ назьвается векторное расслоение $\pi: E \rightarrow B$ над $B$, слоем которого над $\xi$ является факторпространство $E_{\xi}=T_{\xi} M / T_{\xi} B$.

Пусть $p: T_{B} M \rightarrow E$ - естественная проекция. Так как подмногообразие $B$ инвариантно относительно фазового потока поля $X_{H}$, векторное поле $\operatorname{Var}\left(X_{H}\right)$ имеет два инвариантных подмногообразия $T B$ и $T_{B} M$ в $T M$. Можно показать, что векторное поле $\operatorname{Var}\left(X_{H}\right)$ является проектируемым относительно $p$, т.е., на $E$ существует единственное векторное поле $\operatorname{var}_{B}\left(X_{H}\right)$ такое, что

$$
\left(d_{u} p\right) \operatorname{Var}\left(X_{H}\right)(u)=\operatorname{var}_{B}\left(X_{H}\right)(p(u))
$$

для любых $u \in T_{\xi} M$ и $\xi \in B$. Динамическая система $\left(E, B, \operatorname{var}_{B}\left(X_{H}\right)\right)$ называется системой в вариациях поля $X_{H}$ на $B$. Размерность соответствуюшего фазового пространства $E$ (тотального пространства нормального расслоения к листу $B$ ) совпадает с размерностью исходного многообразия, $\operatorname{dim} E=\operatorname{dim} M$. Кроме того, подмногообразие $B \subset E$ (как нулевое сечение расслоения $E$ ) инвариантно относительно фазового потока поля $\operatorname{var}_{B}\left(X_{H}\right)$. Итак, система в вариациях представляет собой естественную линеаризованную модель для исходной гамильтоновой системы $X_{H}$ на $B$. Нас интересует следуюший вопрос: при каких условиях векторное поле $\operatorname{var}_{B}\left(X_{H}\right)$ является гамильтоновым относительно некоторой пуассоновой структуры на $E$ ? Эта задача, например, возникает при изучении (нелинейной) гамильтоновой динамики вблизи (сингулярного) симплектического листа в контексте теории возмущений. В общем случае, процедура линеаризации может разрушить свойство гамильтоновости векторного поля $\operatorname{var}_{B}\left(X_{H}\right)$. В симплектическом случае этот эффект изучался в работах [3]-[5]. В данной статье мы приводим некоторые результаты, касающиеся существования гамильтоновой структуры для $\operatorname{var}_{B}\left(X_{H}\right)$. В основе этих результатов лежит понятие линеаризованной пуассоновой структуры симплектического листа [6], [7].

2. Существование гамильтоновых структур. Рассмотрим дуальное расслоение $E^{*} \subset T_{B}^{*} M$ к $E$, которое будем называть конормальным расслоением к листу $B$, и которое совпадает с ядром морфизма расслоения $\Psi_{B}^{\sharp}: T_{B}^{*} M \rightarrow T_{B} M, \operatorname{ker} \Psi_{B}^{\sharp}=E^{*}$. Тогда каждый слой расслоения $E^{*}$ наследует структуру алгебры Ли $[\cdot, \cdot]_{\text {fib }}$, которая однозначно определяется следующим условием: для произвольных функций $k, \tilde{k}$, заданных на $M$ и постоянных вдоль листа $B$, выполняется соотношение $[\eta, \tilde{\eta}]_{\mathrm{fib}}=\left.d\left(\{k, \tilde{k}\}_{\Psi}\right)\right|_{B}$, 
где $\eta=\left.d k\right|_{B}, \tilde{\eta}=\left.d \tilde{k}\right|_{B}$. Расслоение $E^{*}$ является локально-тривиальным с типичным листом $\mathfrak{g}$, который называется трансверсальной алгеброй Ли симплектического листа $B$. Таким образом, нормальное расслоение пространства $E$ становится локально-тривиальным расслоением Ли-Пуассона над симплектической базой $\left(B, \omega_{B}\right)$. Соответствующая послойная структура Ли-Пуассона индуцирует вертикальньй тензор Пуассона $\Lambda$ на $E$, которьй назьвается линеаризованной трансверсальной пуассоновой структурой исходного тензора Пуассона $\Psi$ на листе $B[8]$, [9].

ОПРЕДЕЛЕНИЕ 2.1. Пуассонова структура П на $E$ назьвается согласованной, если она корректно определена в окрестности нулевого сечения $B$ и удовлетворяет следующим условиям:

(i) $\left(B, \omega_{B}\right)$ является симплектическим листом пуассоновой структуры П;

(ii) линеаризованная трансверсальная пуассонова структура бивекторного поля П на $B$ совпадает с $\Lambda$.

Под трансверсалью $\mathscr{L}$ к $B$ мы будем понимать подрасслоение в $T_{B} M$, являющееся дополнительным к $T B$,

$$
T_{B} M=T B \oplus \mathscr{L}
$$

Пусть $\mathrm{Fl}_{H}^{t}-$ фазовьй поток гамильтонова векторного поля $X_{H}$. Так как $B \subset E-$ это инвариантное подмногообразие для $X_{H}$, дифференциал $d \mathrm{Fl}_{H}^{t}$ действует на $T_{B} M$, оставляя $T B$ инвариантньм.

ТеОрема 2.2. Если фазовый поток гамильтонова векторного поля $X_{H}$ допускает инвариантную трансверсаль $\mathscr{L} \kappa B$,

$$
d \mathrm{Fl}_{H}^{t}(\mathscr{L})=\mathscr{L}
$$

то система в вариациях $\operatorname{var}_{B}\left(X_{H}\right)$ поля $X_{H}$ на $B$ является гамильтоновой относительно некоторой согласованной пуассоновой структуры П на Е и некоторой функиии $F \in C^{\infty}(E)$,

$$
\operatorname{var}_{B}\left(X_{H}\right)=\Pi^{\natural}(d F) .
$$

Ниже для доказательства этой теоремы мы приводим построение согласованной пуассоновой структуры П и функции Гамильтона $F$.

3. Согласованные пуассоновы структуры. Следуя [6], [7], напомним процедуру, которая позволяет, начиная с тройки $(M, \Psi, B)$, построить класс согласованных пуассоновых структур на $E$, параметризованных трансверсалями к $B$.

Имеется естественное расщепление

$$
T_{B} E=T B \oplus E
$$

Фиксируем трансверсаль $\mathscr{L}$ к $B$. Ясно, что $\mathscr{L}$ является подрасслоением $T_{B} E$, которое изоморфно $E$. Ограничение проекции $p$ на слой $\mathscr{L}_{\xi}$ дает изоморфизм на $E_{\xi}$.

Под әкспоненииальным отобраэсением, соответствующим трансверсали $\mathscr{L}$ к $B$, будем понимать диффеоморфизм $\mathbf{f}$ из некоторой окрестности подмногообразия $B$ в $E$ на другую окрестность $B$ в $M$, удовлетворяющий следующим условиям:

$$
\left.\mathbf{f}\right|_{B}=\operatorname{id}_{B}, \quad\left(d_{\xi} \mathbf{f}\right)(e)=p^{-1}(e)
$$


для любого $e \in E_{\xi}$ и $\xi \in B$. В частности, $\left(d_{\xi} \mathbf{f}\right)\left(E_{\xi}\right)=\mathscr{L}_{\xi}$. Экспоненциальное отображение существует в силу теоремы о трубчатой окрестности.

Рассмотрим поднятие $\mathbf{f}^{*} \Psi$ исходной пуассоновой структуры $\Psi$ при экспоненциальном отображении f. Выберем базис $\left\{e^{\sigma}\right\}$ локальных сечений $E^{*}$. Пусть $\left\{e_{\sigma}\right\}-$ дуальный базис расслоения $E$. Рассмотрим координатную систему $\left(\xi^{i}, x^{\sigma}\right)$ на $E$, где $\left(\xi^{i}\right)-$ координаты вдоль $B$, а $\left\{x^{\sigma}\right\}$ - нормальные координаты к $B$, ассоциированные с базисом $\left\{e_{\sigma}\right\}, B=\{x=0\}$. Тогда $\mathbf{f}^{*} \Psi$ является согласованньм тензором Пуассона на $E$. Соответствующие попарные скобки для координатных функций вблизи $B$ имеют вид

$$
\begin{aligned}
\left\{\xi^{i}, \xi^{j}\right\}_{\mathbf{f}^{*} \Psi} & =-\omega^{i j}-\omega^{i s} \mathscr{R}_{s m \nu} \omega^{m j} x^{\nu}+O_{2}, \\
\left\{\xi^{i}, x^{\sigma}\right\}_{\mathbf{f}^{*} \Psi} & =\omega^{i j} \theta_{j \nu}^{\sigma} x^{\nu}+O_{2}, \\
\left\{x^{\alpha}, x^{\beta}\right\}_{\mathbf{f}^{*} \Psi} & =\lambda_{\nu}^{\alpha \beta} x^{\nu}+O_{2} .
\end{aligned}
$$

Здесь $\omega^{i s}(\xi) \omega_{s j}(\xi)=\delta_{j}^{i}, \quad\left(\omega_{s j}\right)-$ коэффиициенты симплектической формы (1.3), а $\lambda_{\nu}^{\alpha \beta}$, $\theta_{\nu j}^{\sigma}, \mathscr{R}_{s m \nu}-$ гладкие функции на $B$. Символ $O_{k}$ обозначает член порядка $k$ в формальном разложении функции в ряд Тейлора в точке $x=0$. Отметим, что функции $\lambda_{\nu}^{\alpha \beta}=\lambda_{\nu}^{\alpha \beta}(\xi)$ являются структурными константами скобок Ли на слое $E$ относительно базиса $\left\{e^{\sigma}(\xi)\right\}$.

Через $\Omega^{k}(B, E)$ будем обозначать пространство векторно-значных $k$-форм на $B$ со значениями в пространстве гладких сечений пространства $E$. В частности, $\Omega^{0}(B, E)=$ $C^{\infty}(B ; E)$ является пространством векторно-значных функций на $E$. Введем матрично-значную 1 -форму $\theta^{\mathscr{L}}=\left(\theta_{\beta}^{\alpha}\right)$ и векторно-значную 2 -форму $\mathscr{R}^{\mathscr{L}}=\left(\mathscr{R}_{\sigma}\right) \in \Omega^{2}\left(B, E^{*}\right)$ с компонентами

$$
\theta_{\beta}^{\alpha}=\theta_{i \beta}^{\alpha}(\xi) d \xi^{i}, \quad \mathscr{R}_{\sigma}=\frac{1}{2} \mathscr{R}_{i j \sigma}(\xi) d \xi^{i} \wedge d \xi^{j}
$$

относительно базиса $\left\{e^{\alpha}\right\}$. Можно показать [10], что существует линейная связность $\nabla^{\mathscr{L}}$ на $E$, для которой форма связности относительно базиса $\left\{e_{\sigma}\right\}$ задается в точности 1-формой $\theta^{\mathscr{L}}$. Важное наблюдение состоит в следующем: параллельный перенос $\nabla^{\mathscr{L}}$ сохраняет послойную структуру Ли-Пуассона линейной связности $E$. Кроме того, форма кривизны связности $\nabla^{\mathscr{L}}$ выражается через $\mathscr{R}^{\mathscr{L}}$ следуюшим образом:

$$
\operatorname{Curv}^{\nabla^{\mathscr{L}}}=d \theta^{\mathscr{L}}+\theta^{\mathscr{L}} \wedge \theta^{\mathscr{L}}=-\operatorname{ad}^{*} \circ \mathscr{R}^{\mathscr{L}} \text {. }
$$

Здесь $\mathrm{ad}^{*}$ - оператор коприсоединенного представления, действующий на слоях расслоения $E$. Через

$$
\operatorname{hor}_{i} \stackrel{\text { def }}{=} \frac{\partial}{\partial \xi^{i}}-\theta_{i \nu}^{\sigma}(\xi) x^{\nu} \frac{\partial}{\partial x^{\sigma}}
$$

обозначим горизонтальньй лифт базисного векторного поля $\partial / \partial \xi^{i}, i=1, \ldots, \operatorname{dim} B$. Также введем скалярную 2-форму $\mathscr{F}=\frac{1}{2} \mathscr{F}_{i j}(\xi, x) d \xi^{i} \wedge d \xi^{j}$ на $E$ с коэффициентами

$$
\mathscr{F}_{i j}(\xi, x) \stackrel{\text { def }}{=} \omega_{i j}(\xi)-x^{\nu} \mathscr{R}_{i j \nu}(\xi) \text {. }
$$

Заметим, что $\mathscr{F}_{i j}(\xi, 0)=\omega_{i j}(\xi)$ и, следовательно, $\operatorname{det}\left[\mathscr{F}_{i j}(\xi, 0)\right] \neq 0 \quad \forall \xi \in B$. Таким образом, 2-форма $\mathscr{F}$ является невырожденной в открытой области

$$
\mathscr{N}=\left\{(\xi, x) \in E \mid \operatorname{det}\left[\mathscr{F}_{i j}(\xi, x)\right] \neq 0\right\},
$$


содержащей $B$. Элементы обратной к $\left[\mathscr{F}_{i j}\right]$ матрицы обозначим через $\mathscr{F}^{i j}=\mathscr{F}^{i j}(\xi, x)$, $\mathscr{F}^{i s} \mathscr{F}_{s j}=\delta_{j}^{i}$. Введем бивекторное поле $\Pi_{\mathscr{L}}$ на $\mathscr{N} \subseteq E$, ассоциированное с данными $\left(\nabla^{\mathscr{L}}, \mathscr{R}^{\mathscr{L}}\right)$, которые задаются соотношением

$$
\Pi_{\mathscr{L}} \stackrel{\text { def }}{=}-\frac{1}{2} \mathscr{F}^{i j} \operatorname{hor}_{i} \wedge \operatorname{hor}_{j}+\Lambda .
$$

Здесь бивекторное поле $\Lambda$ на $E$ задано соотношением

$$
\Lambda=\frac{1}{2} \lambda_{\nu}^{\alpha \beta}(\xi) x^{\nu} \frac{\partial}{\partial x^{\alpha}} \wedge \frac{\partial}{\partial x^{\beta}}
$$

и определяет линеаризованную трансверсальную пуассонову структуру тензорного поля $\Psi$ на $B$.

ПРЕДЛОЖЕНИЕ 3.1 [8]. Для любой трансверсали $\mathscr{L}$ векторное поле $\Pi_{\mathscr{L}}$ в (3.7) задает согласованный тензор Пуассона на $\mathscr{N} \subseteq E$.

Заметим, что пуассонова структура $\Pi_{\mathscr{L}}$ не зависит от выбора трансверсали $\mathscr{L}$ с точностью до изоморфизма в окрестности $B$ (см. [6], [7]). Этот факт ведет к понятию линеаризованной пуассоновой структуры данного симплектического листа $B$.

Пусть задано линейное векторное поле $\mathscr{V}_{f}$ на $E$, которое проектируется на гамильтоново векторное поле $v_{f}$ на $(B, \omega)$,

$$
\mathscr{V}_{f}=v_{f}^{i}(\xi) \frac{\partial}{\partial \xi^{i}}+V_{\nu}^{\alpha}(\xi) x^{\nu} \frac{\partial}{\partial x^{\alpha}}
$$

где $V=\left(V_{\nu}^{\alpha}(\xi)\right)$ - матрично-значная функция на $B$.

ПРЕДЛОЖЕНИЕ 3.2. Линейное векторное поле $\mathscr{V}$ является гамильтоновым относительно пуассоновой структуры $\Pi_{\mathscr{L}}$ и послойно линейной функиии $\digamma=\pi^{*} f-$ $\langle x, \eta\rangle, \eta \in C^{\infty}(B, E)$, тогда и только тогда, когда пара $(\eta, V)$ удовлетворяет следующим уравнениям на $B$ :

$$
\begin{gathered}
d \eta-\left(\theta^{\mathscr{L}}\right)^{T} \eta=-\mathbf{i}_{v_{f}} \mathscr{R}^{\mathscr{L}} \\
V=-\left(\mathbf{i}_{v_{f}} \theta^{\mathscr{L}}+\operatorname{ad}^{*} \circ \eta\right) .
\end{gathered}
$$

Здесь $\mathbf{i}_{v_{f}}$ обозначает внутреннее произведение векторного поля $v_{f}$ и дифференциальной формы на $B$.

4. Инвариантные трансверсали и динамическое кручение. Пусть $\mathbf{f}$ - экспоненциальное отображение, ассоциированное с трансверсалью $\mathscr{L}$. Рассмотрим поднятие пуассоновой структуры $\mathbf{f}^{*} \Psi$ на $E$. Симплектический лист $(B, \omega)$ является также симплектическим листом для $\mathbf{f}^{*} \Psi$, чье нормальное расслоение отождествляется с $E$. Разлагая гамильтонову систему $\left(E, \mathbf{f}^{*} \Psi, \mathbf{f}^{*} H=H \circ \mathbf{f}\right)$ в ряд Тейлора в точке $x=0$ и используя соотношения (3.2)-(3.4), получаем

$$
\begin{aligned}
\frac{d \xi^{i}}{d t} & =\omega^{i s}(\xi) \frac{\partial f(\xi)}{\partial \xi^{s}}+\Upsilon_{\nu}^{i}(\xi) x^{\nu}+O_{2} \\
\frac{d x^{\sigma}}{d t} & =\left[\lambda_{\nu}^{\sigma \beta}(\xi) \eta_{\beta}(\xi)-\theta_{j \nu}^{\sigma}(\xi) \omega^{j s}(\xi) \frac{\partial f(\xi)}{\partial \xi^{s}}\right] x^{\nu}+O_{2}
\end{aligned}
$$


Здесь $f(\xi)=\mathbf{f}^{*} H(\xi, 0)$ и

$$
\eta_{\nu}(\xi)=-\frac{\partial\left(\mathbf{f}^{*} H\right)}{\partial x^{\nu}}(\xi, 0),
$$

а гладкие функции $\Upsilon_{\nu}^{i}$ на $B$ заданы соотношениями

$$
\Upsilon_{\nu}^{i} \stackrel{\text { def }}{=}-\omega^{i j} \frac{\partial \eta_{\nu}}{\partial \xi^{j}}+\omega^{i j} \theta_{j \nu}^{\alpha} \eta_{\alpha}+\omega^{i s} \mathscr{R}_{s m \nu} \omega^{m j} \frac{\partial f}{\partial \xi^{j}} .
$$

$\mathrm{C}$ помощью функций $\eta_{\nu}$ определим вектор-функцию функции $\eta^{\mathscr{L}}=\eta_{\nu}(\xi) \otimes e^{\nu}(\xi)$ на $B$ (глобальное сечение расслоения $E^{*}$ ). Кроме того, можно показать, что $\Upsilon_{\nu}^{i}$ задают векторное поле

$$
\Upsilon^{\mathscr{L}}=\Upsilon_{\nu}^{i}(\xi) x^{\nu} \frac{\partial}{\partial \xi^{i}}
$$

на $E$, которое можно назвать кручением фазового потока поля $X_{\mathbf{f}^{*} H}$ относительно разложения (2.1). Зануление кручения $\Upsilon^{\mathscr{L}}=0$ имеет следующую интерпретацию: трансверсаль инвариантна относительно $d \mathrm{Fl}_{\mathbf{f}^{*} H}^{t}$. Ниже мы увидим, что $\eta^{\mathscr{L}}$ и $\Upsilon^{\mathscr{L}}$ зависят от выбора $\mathscr{L}$.

Теперь из соотношения (4.2) следует, что система в вариациях гамильтонова векторного поля $X_{\mathbf{f}^{*} H}$ на $B$ имеет вид

$$
\begin{aligned}
& \frac{d \xi}{d t}=v_{f}, \\
& \frac{d x}{d t}=-\left(\operatorname{ad}_{\eta}^{*}+\mathbf{i}_{v_{f}} \theta\right) x .
\end{aligned}
$$

Соответственно, векторное поле можно представить в виде

$$
\operatorname{var}_{B}\left(X_{\mathbf{f}^{*} H}\right)=\operatorname{hor}_{v_{f}}-\left\langle\operatorname{ad}_{\eta}^{*} x, \frac{\partial}{\partial x}\right\rangle .
$$

Здесь hor $_{v_{f}}$ является горизонтальным лифтом гамильтонова векторного поля $v_{f}$ относительно связности $\nabla^{\mathscr{L}}$.

Теперь рассмотрим две трансверсали $\mathscr{L}$ и $\widetilde{\mathscr{L}}$ к $B$. Пусть $l: T_{B} M \rightarrow \widetilde{\mathscr{L}}$ - проекция вдоль $T B$ в соответствии с разложением (3.1). Задавая базис $\left\{n_{\sigma}\right\}$ (локальных) сечений

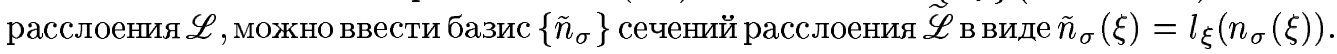
Тогда имеем

$$
\tilde{n}_{\sigma}(\xi)=n_{\sigma}+u_{\sigma}(\xi)
$$

где $u_{\sigma}$ - некоторые векторные поля на $B, u_{\sigma}(\xi) \in T_{\xi} B$. Используя эти векторные поля и симплектическую 2-форму $\omega$ на $B$, определим векторно-значную 1 -форму $\varrho \in \Omega^{1}\left(B, E^{*}\right)$ следуюшим образом:

$$
\varrho \stackrel{\text { def }}{=}-\left(\mathbf{i}_{u_{\nu}} \omega\right) \otimes e^{\nu},
$$

или, в координатном виде, как $\varrho_{i \nu}=\omega_{i j} u_{\nu}^{j}$. Так как $\omega$ невырожденная форма, при фиксированном $\mathscr{L}$ формула (4.9) задает однозначное соответствие между множеством всех 
трансверсалей к $B$ и пространством $\Omega^{1}\left(B, E^{*}\right)$ векторно-значных 1-форм на $B$. Непосредственные вычисления показьвают, что данные, соответствующие $\mathscr{L}$ и $\widetilde{\mathscr{L}}$, удовлетворяют соотношениям

$$
\begin{aligned}
& \nabla^{\widetilde{L}}=\nabla^{\mathscr{L}}-\operatorname{ad}^{*} \circ \varrho, \\
& \mathscr{R}^{\widetilde{L}}=\mathscr{R}^{\mathscr{L}}+\left(\nabla^{\mathscr{L}}\right)^{*} \varrho^{\mathscr{L}}+\frac{1}{2}\left[\varrho^{\mathscr{L}} \wedge \varrho^{\mathscr{L}}\right] .
\end{aligned}
$$

Кроме того,

$$
\eta_{\sigma}^{\widetilde{\mathscr{L}}}=\eta_{\sigma}^{\mathscr{L}}-L_{u_{\sigma}} f
$$

Из этих соотношений вытекает следующее утверждение.

ПРЕДЛОЖЕНИЕ 4.1. Система в вариациях, соответствующая гамильтоновой системе $X_{\mathbf{f}^{*} \text { н }}$ не зависит от выбора экспоненциального отображсения $\mathbf{f}$,

$$
\operatorname{var}_{B}\left(X_{\mathbf{f}^{*} H}\right)=\operatorname{var}_{B}\left(X_{H}\right) .
$$

Сравнивая (3.8) и (4.7) с (4.4), мы видим, что условие $\Upsilon^{\mathscr{L}}=0$ эквивалентно уравнениям $(3.8),(3.9)$ для $\eta^{\mathscr{L}}$ в $(4.3)$. Таким образом, из предложения 3.2 следует основной результат нашей статьи.

ТЕОремА 4.2. Пусть существует трансверсаль $\mathscr{L}$, которая инвариантна относительно $d \mathrm{Fl}_{H}^{t}$. Пусть $\Pi^{\mathscr{L}}$ - соответствующая пуассонова структура в (3.7). Тогда векторное поле $\operatorname{var}_{B}\left(X_{H}\right)$ является гамильтоновым относительно П ${ }^{\mathscr{L}} u$ функиии

$$
F^{\mathscr{L}}(\xi, x)=f(\xi)-\left\langle x, \eta^{\mathscr{L}}(\xi)\right\rangle .
$$

В частном случае, анализируя уравнения (3.8), (3.9), можно вьвести следующий критерий.

ТЕОремА 4.3. Если трансверсальная алгебра Ли g симплектического листа В является полупростой, то существование $X_{H}$-инвариантной трансверсали является необходимым и достаточным условием для того, чтобы система в вариация $\operatorname{var}_{B}\left(X_{H}\right)$ была гамильтоновой в классе согласованных гамильтоновых структур на $E$.

С помощью этого критерия можно описать возможные препятствия к существованию гамильтоновой структуры в следующей простой ситуации.

ПримеР 4.4. Пусть $B=(a, b) \times \mathbb{S}^{1}$ - двумерньй цилиндр с введенной на нем канонической симплектической структурой $\omega=d s \wedge d \tau$, где $s \in(a, b)$ и $\tau(\bmod 2 \pi)$ - угловая координата на окружности $\mathbb{S}^{1}=\mathbb{R} / 2 \pi \mathbb{Z}$. Рассмотрим циклические пуассоновы скобки на $\mathbb{R}^{3}$, связанные с алгеброй Ли so(3),

$$
\left\{x^{1}, x^{2}\right\}=x^{3}, \quad\left\{x^{2}, x^{3}\right\}=x^{1}, \quad\left\{x^{3}, x^{1}\right\}=x^{2} .
$$

Рассмотрим $M=(a, b) \times \mathbb{S}^{1} \times \mathbb{R}^{3}$ как прямое произведение двух пуассоновых многообразий. Ясно, что $B=(a, b) \times \mathbb{S}^{1}$ является симплектическим листом многообразия $M$. Рассмотрим гамильтонову систему на $M$, соответствующую функции

$$
H=1-\langle x, \phi(s, \tau)\rangle+O_{2},
$$


где $\phi(s, \tau)=\phi(s, \tau+2 \pi)$ - гладкая вектор функция, $2 \pi$-периодическая по $\tau$. Соответствующая система в вариациях гамильтонового поля $X_{H}$ на листе $B$ имеет вид

$$
\begin{gathered}
\dot{s}=0, \quad \dot{\tau}=1, \\
\frac{d \mathbf{x}}{d \tau}=\phi(s, \tau) \times \mathbf{x} .
\end{gathered}
$$

Ясно, что (4.14) представляет собой однопараметрическое семейство периодических линейных систем на $\mathbb{R}^{3}$. Пусть $M(s)$ - соответствующая матрища монодромии, гладко зависящая от $s$. Тогда можно показать, что система (4.13), (4.14) допускает согласованную гамильтонову структуру тогда и только тогда, когда $M(s)$ удовлетворяет уравнению типа Лакса

$$
\frac{d \mathscr{M}(s)}{d s}=[\mathscr{M}(s), \mathbb{A} \circ \boldsymbol{\mu}(s)]
$$

для некоторой гладкой вектор-функции $\boldsymbol{\mu}(s)$. Здесь $\mathbb{A} \circ \boldsymbol{\mu}(s)$ обозначает $(3 \times 3)$ кососимметричную матрищу векторного произведения на $\mathbb{R}^{3}$. Отсюда следует, что сnектр $\mathscr{M}(s)$ не зависит от $s$ (т.е. монодромия обладает свойством изоспектральной деформачии). В противном случае, когда $\operatorname{spec} \mathscr{M}(s)$ меняется вместе с изменением $s$, система (4.13), (4.14) не допускает согласованную гамильтонову структуру.

Автор благодарит М. В. Карасева и Рубена Флореса Эспинозу за полезные обсуждения различных аспектов данной работы.

\section{СПИСОК ЦИТИРОВАННОЙ ЛИТЕРАТУРЫ}

[1] Marsden J., Ratiu T., Rangel G. Symplectic connections and the linearization of hamiltonian systems // Proc. Roy. Soc. Edinburgh. Sect. A. 1991. V. 117. P. 329-380.

[2] Marsden J.E., Ratiu T.S. Introduction to Mechanics and Symmetry. New York: Springer-Verlag, 1994.

[3] Karasev M. V., Vorobjev Yu. M. Adapted connections, Hamiltonian dynamics, geometric phases, and quantization over isotropic submanifolds // Amer. Math. Soc. Transl. 1998. V. 187. № 2. P. 203-326.

[4] Flores Espinoza R., Vorobiev Yu. M. Hamiltonian formalism for fiberwise linear systems // Bol. Soc. Mat. Mexicana. 2000. V. 6. № 3. P. 213-234.

[5] Vorobiev Yu. Hamiltonian equations of the first variation equations // Mathematics. 2000. V. 191. № 4. P. 447-502.

[6] Vorobjev Yu. Coupling tensors and Poisson geometry near a single symplectic leaf // Lie Algebroids. V. 54. Warszawa: Banach Center Publ., 2001. P. 249-274.

[7] Воробьев Ю. М. О линеаризованных пуассоновых структурах // Матем. заметки. 2001. V. 70. № 4. P. 486-493.

[8] Weinstein A. The local structure of Poisson manifolds // J. Diff. Geom. 1983. V. 18. P. 523-557.

[9] Cannas da Silva A., Weinstein A. Geometric Models for Noncommutative Algebras. Berkeley Math. Lecture Notes. Providence, RI: Amer. Math. Soc., 1999.

[10] Karasev M. V., Vorobjev Yu. M. Deformations and cohomology of Poisson manifolds // Lecture Notes in Math. V. 1453. Berlin: Springer-Verlag, 1990. P. 271-289. 